\title{
Liver transplantation and concomitant prostate cancer: is it feasible?
}

\begin{abstract}
Nowadays liver transplantation is the standard treatment for chronic and acute liver failure, with excellent patient and graft survival rates, which both have improved through the years. Liver transplant (LT) candidates must undergo a specific and thorough transplant evaluation including several tests and screening for extra hepatic malignancies. Screening for prostate cancer is recommended in all male candidates older than 50 years. Although LT is precluded in candidates with concomitant neoplasms other than biliary or liver, early prostate cancer has very good outcomes with curative treatments. However, international guidelines contraindicate LT in patients with extrahepatic malignancies. We therefore report a case of 65 years old male with primary biliary cholangitis (PBC), who underwent a first LT in 2003. The patient latter developed progressive renal failure and loss of liver graft due to PBC recurrence. He was then evaluated for simultaneous liver-kidney (SLK) transplantation. During screening tests he was diagnosed with non-invasive prostate adenocarcinoma with no metastatic lesions (Stage II). Balancing risks and benefits before transplant, we decided to avoid prostatectomy and treat the patient with hormonal therapy (Goserelin). The patient underwent SKL transplantation successfully, continued with hormonal therapy and received local radiotherapy the first year after transplant without complications. We report here a unique case of combined liver and renal transplant with active prostate cancer, who received only hormonal therapy before transplantation with absence of progression while on immunosuppression.
\end{abstract}

Keywords: liver transplant, renal transplant, prostatic cancer, prostatic specific antigen
Volume 8 Issue I - 2017

\author{
Josefina Pages,' Carla Colaci,' Manuel \\ Mendizabal,' Federico Piñero,' Cristina \\ Alonso,' Ariel Gonzalez Campa a,' Martin \\ Fauda,' Alejandro Nolazco, ${ }^{2}$ Gustavo \\ Podesta,' Marcelo Silva' \\ 'Liver Transplant and Hepatobiliary Unit, Austral University, \\ Argentina \\ ${ }^{2}$ Department of Urology, Austral University, Argentina
}

Correspondence: Josefina Pagés, Hepatology and Liver Transplant Unit, Hospital Universitario Austral, Av. Presidente Perón 1500, (BI629HJ) Pilar, Buenos Aires, Argentina, Tel +54 0230448 2000, Fax +540230 4482236 ,

Email jpages@cas.austral.edu.ar

Received: September 30, 2017 | Published: November 20, 2017
Abbreviations: LT, liver transplant; OLT, orthotopic liver transplantation; RT, renal transplant; KT, kidney transplant; LTR, liver transplant recipients; RTR, renal transplant recipients; PSA, prostatic specific antigen; PBC, primary biliary cholangitis; CT, computed tomography; SKL, simultaneous liver-kidney; SOT, solid organ transplantation; NCCN, national comprehensive cancer network

\section{Introduction}

Thomas Starz made the first liver transplant (LT) in 1967. Since then, advances in several fields, such us improvement in surgical techniques, immunosuppressive agents, management of postoperative complications, have made LT the only curative treatment for patients with end stage liver disease and acute liver failure with a significant increase in survival. ${ }^{1}$ Survival rates ranges from $90 \%$ to $70 \%$ at one and five years, respectively. ${ }^{2}$ Evaluating and selecting a good recipient for solid organ transplantation (SOT) requires a thoruogh examination by diferent specialists to exclude potential comorbidities that might contraindicate the procedure. Screening tests, according to age and risk factors, must include the exclusion of any disseminated cancer or local aggressive malignancy. ${ }^{3,4}$ In order to minimize the chance of cancer relapse after LT, a 5-year disease free interval is recommended in those patients with history of extra hepatic neoplasm. In male candidates with 50 years or older, prostate cancer screening is strongly recommended, ${ }^{5}$ including prostate specific antigen (PSA) testing and rectal examination. ${ }^{5,6}$ Prostate cancer treatment varies according to the disease stage. Prostatectomy, radiotherapy and hormonal therapy are temain as the best treatment options. ${ }^{7}$ We therefore report a case of a male patient with stage II prostate cancer in whom simultaneous liver-kidney (SKL) transplantation was performed without achieving complete curative treatment before surgery.

\section{Case presentation}

A 65-year old gentleman with history of end-stage liver disease secondary to primary biliary cholangitis (PBC) underwent a first orthotopic liver transplantation (OLT) in 2003. Post-OLT immunosuppression was based on cyclosporine A, mycophenolate mofetil and meprednisone. Two years after OLT, the patient developed progressive renal failure secondary to calcineurin inhibitors and graft failure with clinically significant portal hypertension due to PBC recurrence. The patient was evaluated for SLK transplantation. Screening tests for malignant neoplasms according to his age were performed. PSA was $10 \mathrm{mg} / \mathrm{ml}$, a 45 -gr prostate without any nodule was observed on the ultrasound. Rectal examination revealed an indurated prostate with no nodules. A multifoci prostate biopsy was then performed (fourteen samples) and five of them were positive for adenocarcinoma; Gleason Score was $7(4+3)$. No metastatic lesions were found in chest-computed tomography (CT) or in complete bone scans. Prostate cancer was classified as clinically localized, NCCN intermediate risk, Stage II. The patient had a Child Pugh C classification with high risk of complications in order to perform a prostatectomy. According to the patient's clinical condition, the best approach was discussed by a multidisciplinary team integrated by hepatologists, nephrologists, oncologists, urologists and surgeons. It was then decided to include the patient in the waiting list for SLK transplantation and to treat prostate cancer with androgen deprivation therapy. The patient was placed on hormonal treatment with goserolin. In September 2011 the patient underwent SLK transplantation. He received induction therapy with basiliximab $20 \mathrm{mg}$ (day 0 and 4 ) and methilprednisolone 1 gr during the an hepatic phase. The patient continued with standard immunosuppression starting delayed tacrolimus on day 4 with mycophenolate mofetil (360 bid). 
Corticosteroids were tapper off after 3 months. Immeadiate posttransplant period was uneventful, in July 2012 he started hormonal treatment in combination with local radiotherapy (43 sessions). Serum PSA levels were $<0,015$ by the end of this treatment, and 0,06 one year later. Six years after the second transplant there is still no evidence of prostatic cancer recurrence.

\section{Discussion}

To the best of our knowledge this is first successful case published of a SOT performed in a patient with known concomitant prostate cancer who did not received complete curative treatment before transplantation. It is important to mention several issues, first this is a clinical case about a liver transplanted patient due to PBC that developed prostate cancer under immunosuppressive regimen. Second, this is also a case of liver and renal transplant with active prostate cancer at the time the procedure was done. Undergoing any SOT in candidates with malignant extrahepatic cancer without prior treatment is controversial. Most guidelines suggest a 5-year disease free interval. In patients with intermediate stage prostate cancer there are two possible treatments; prostatectomy or radiotherapy (beam source or brachytherapy) combined with androgen deprivation therapy. Studies have demonstrated that 4 to 6 months of hormonal therapy improves survival in patients with intermediate or high-risk prostate cancer. On the other hand there is no difference between radiotherapy and other treatment modalities such as prostatectomy and brachytherapy. ${ }^{7}$ However, our patient presented active prostate cancer previous to SLK and he was only treated with hormonal therapy and no prostatectomy nor radiotherapy before the surgery.

Kocak et al. ${ }^{8}$ described a case of a patient with prostate cancer that underwent kidney transplant only 3 months after being treated with prostatatectomy. Nevertheless, the patient received treatment before surgery and there is no further information of the post transplant outcomes. ${ }^{8}$ Woodle et al. ${ }^{9}$ analysed a large cohort of patients who presented prostate cancer prior to SOT. Only 3 of them underwent OLT, 77 received a kidney transplant, and there were no cases of SLK transplant. The median time to recurrence was 10.6 months after transplantation and the median survival time was 49.2 months. Prostate cancer recurrence for stage II was $15.9 \%$ relatively low, but rates of recurrences increased if the disease extended beyond the prostate. However, it is important to mention that the investigators didn't have information on the patient's serum PSA and Gleason score, which is relevant data when it comes to risk stratification in prostate cancer. On the other hand, the study included renal transplanted patients with prostate cancer prior to transplantation, still there is no information regarding prostate cancer outcomes when treated after transplantation. ${ }^{9}$ It has been described that immunosuppression can promote cancer development or progression after SOT. However, the specific effect of immunosuppressive drugs on prostate cancer is still unknown, with a lack of robust evidence suggesting any deleterious effect on cancer progression. ${ }^{8-11}$ Considering all these information, the natural history of prostate cancer under immunosuppressive drugs and in SOT is controversial. There is no information regarding management of prostate cancer in SLK transplantation. Thus, treatment of prostate cancer in OLT recipients should be the same as in general population, surgery or radiation. ${ }^{11}$

\section{Conclusion}

In conclusion, although most of the non-hepatobiliary cancers would be a contraindication for SOT we report a case of SLK transplant candidate with prostate cancer who received curative treatment after transplantation. This may imply that if the patient has an early or intermediate stage prostate cancer associated with other comorbidities that might contraindicate prostatectomy, hormonal therapy arises as a potential alternative to control cancer progression before transplantation.

\section{Acknowledgments}

We thank LALREAN for its commitment and assistance with editing of this research.

\section{Conflicts of interest}

The authors of this manuscript have no conflicts of interest. This research received no specific grant from any funding agency in the public, commercial, or non-profit sectors.

\section{References}

1. Roberts MS, Angus DC, Bryce CL, et al. Survival after liver transplantation in the United States: a disease-specific analysis of the UNOS database. Liver transplantation. 2004;10(7): 886-897.

2. Moreno R, Berenguer M. Post-liver transplantation medical complications. Ann Hepatol. 2006;5(2):77-85.

3. Burra P, Burroughs A, et al. EASL clinical practice guidelines: liver transplantation. J Hepatol. 2016;64(2):433-485.

4. Martin P, Di Martini A, Feng S, et al. Evaluation for liver transplantation in adults: 2013 practice guideline by the American Association for the Study of Liver Diseases and the American Society of Transplantation. Hepatology. 2014;59(3):1144-1165.

5. Humphrey PA, Schuz J. Cancers of the male reproductive organs. World Cancer Report. Lyon, France. World Health Organization; 2014. pp. 453-464.

6. Smith RA, von Eschenbach AC, Wender R, et al. American Cancer Society guidelines for the early detection of cancer: update of early detection guidelines for prostate, colorectal, and endometrial cancers: Also: update 2001-testing for early lung cancer detection. CA Cancer J Clin. 2001;51(1):38-75.

7. Nguyen PL, Aizer A, Assimos DG, et al. ACR Appropriateness Criteria Definitive External-Beam Irradiation in stage $\mathrm{T} 1$ and $\mathrm{T} 2$ prostate cancer. Am J Clin Oncol. 2014;37(3):278-288.

8. Kocak B, Bilen CY, Dilek M, et al. Is Localized Prostate Cancer an Obstacle for an Immediate Consideration for Renal Transplantation? A Case Report. Transplant Proc. 2009;41(5):1961-1962.

9. Woodle ES, Gupta M, Buell JF, et al. Prostate cancer prior to solid organ transplantation: the Israel Penn International Transplant Tumor Registry experience. Transplant Proc. 2005;37(2):958-959.

10. Breyer BN, Whitson JM, Freise CE, et al. Prostate cancer screening and treatment in the transplant population: current status and recommendations. J Urol. 2009;181(5):2018-2026.

11. Tillou X, Chiche L, Guleryuz K, et al. Prostate carcinoma in liver transplant recipients: Think about it!. In Urologic Oncology: Seminars and Original Investigations. 2015;33(6):265. 